\title{
A Note on Solutions of Linear Systems
}

\author{
Branko Malešević, ${ }^{1}$ Ivana Jovović, ${ }^{1}$ Milica Makragić, ${ }^{1}$ and Biljana Radičićc ${ }^{2}$ \\ ${ }^{1}$ Faculty of Electrical Engineering, University of Belgrade, Bulevar kralja Aleksandra 73, 11000 Belgrade, Serbia \\ ${ }^{2}$ Faculty of Civil Engineering, University of Belgrade, Bulevar kralja Aleksandra 73, 11000 Belgrade, Serbia
}

Correspondence should be addressed to Ivana Jovović; ivana@etf.rs

Received 29 April 2013; Accepted 5 July 2013

Academic Editors: A. Jaballah, E. W. H. Lee, and F. Marko

Copyright (c) 2013 Branko Malešević et al. This is an open access article distributed under the Creative Commons Attribution License, which permits unrestricted use, distribution, and reproduction in any medium, provided the original work is properly cited.

We will consider Rohde's general form of $\{1\}$-inverse of a matrix $A$. The necessary and sufficient condition for consistency of a linear system $A x=c$ will be represented. We will also be concerned with the minimal number of free parameters in Penrose's formula $x=A^{(1)} c+\left(I-A^{(1)} A\right) y$ for obtaining the general solution of the linear system. These results will be applied for finding the general solution of various homogenous and nonhomogenous linear systems as well as for different types of matrix equations.

\section{Introduction}

In this paper, we consider nonhomogeneous linear system in $n$ variables

$$
A x=c,
$$

where $A$ is an $m \times n$ matrix over the field $\mathbb{C}$ of rank $a$ and $c$ is an $m \times 1$ matrix over $\mathbb{C}$. The set of all $m \times n$ matrices over the complex field $\mathbb{C}$ will be denoted by $\mathbb{C}^{m \times n}, m, n \in \mathbb{N}$. The set of all $m \times n$ matrices over the complex field $\mathbb{C}$ of rank $a$ will be denoted by $\mathbb{C}_{a}^{m \times n}$. For simplicity of notation, we will write $A_{i \rightarrow}\left(A_{\downarrow j}\right)$ for the $i$ th row (the $j$ th column) of the matrix $A \in$ $\mathbb{C}^{m \times n}$.

Any matrix $X$ satisfying the equality $A X A=A$ is called $\{1\}$-inverse of $A$ and is denoted by $A^{(1)}$. The set of all $\{1\}$ inverses of the matrix $A$ is denoted by $A\{1\}$. It can be shown that $A\{1\}$ is not empty. If the $n \times n$ matrix $A$ is invertible, then the equation $A X A=A$ has exactly one solution $A^{-1}$, so the only $\{1\}$-inverse of the matrix $A$ is its inverse $A^{-1}$; that is, $A\{1\}=\left\{A^{-1}\right\}$. Otherwise, $\{1\}$-inverse of the matrix $A$ is not uniquely determined. For more information about $\{1\}$ inverses and various generalized inverses, we recommend Ben-Israel and Greville [1] and Campbell and Meyer [2].

For each matrix $A \in \mathbb{C}_{a}^{m \times n}$ there are regular matrices $P \in$ $\mathbb{C}^{n \times n}$ and $Q \in \mathbb{C}^{m \times m}$ such that

$$
Q A P=E_{a}=\left[\begin{array}{c|c}
I_{a} & 0 \\
\hline 0 & 0
\end{array}\right],
$$

where $I_{a}$ is $a \times a$ identity matrix. It can be easily seen that every $\{1\}$-inverse of the matrix $A$ can be represented in the form

$$
A^{(1)}=P\left[\begin{array}{c|c}
I_{a} & U \\
\hline V & W
\end{array}\right] Q,
$$

where $U=\left[u_{i j}\right], V=\left[v_{i j}\right]$, and $W=\left[w_{i j}\right]$ are arbitrary matrices of corresponding dimensions $a \times(m-a),(n-a) \times a$, and $(n-a) \times(m-a)$ with mutually independent entries; see Rohde [3] and Perić [4].

We will generalize the results of Urquhart [5]. Firstly, we explore the minimal numbers of free parameters in Penrose's formula

$$
x=A^{(1)} c+\left(I-A^{(1)} A\right) y
$$

for obtaining the general solution of the system (1). Then, we consider relations among the elements of $A^{(1)}$ to obtain the general solution in the form $x=A^{(1)} c$ of the system (1) for $c \neq 0$. This construction has previously been used by Malešević and Radičić [6] (see also [7] and [8]). At the end of this paper, we will give an application of this results to the matrix equation $A X B=C$.

\section{The Main Result}

In this section, we indicate how a technique of an $\{1\}$-inverse may be used to obtain the necessary and sufficient condition 
for an existence of a general solution of a nonhomogeneous linear system.

Lemma 1. The nonhomogeneous linear system (1) has a solution if and only if the last $m-a$ coordinates of the vector $c^{\prime}=Q c$ are zeros, where $Q \in \mathbb{C}^{m \times m}$ is regular matrix such that (2) holds.

Proof. The proof follows immediately from KroneckerCapelli theorem. We provide a new proof of the lemma by using the $\{1\}$-inverse of the system matrix $A$. The system (1) has a solution if and only if $c=A A^{(1)} c$; see Penrose [9]. Since $A^{(1)}$ is described by (3), it follows that

$$
A A^{(1)}=A P\left[\begin{array}{c|c}
I_{a} & U \\
\hline V & W
\end{array}\right] Q=Q^{-1}\left[\begin{array}{c|c}
I_{a} & U \\
\hline 0 & 0
\end{array}\right] Q .
$$

Hence, we have the following equivalences:

$$
\begin{aligned}
c=A A^{(1)} c & \Longleftrightarrow\left(I-A A^{(1)}\right) c=0 \\
& \Longleftrightarrow\left(Q^{-1} Q-Q^{-1}\left[\begin{array}{c|c}
I_{a} & U \\
\hline 0 & 0
\end{array}\right] Q\right) c=0 \\
& \Longleftrightarrow Q^{-1}\left[\begin{array}{l|l}
0 & -U \\
\hline 0 & I_{n-a}
\end{array}\right] \underbrace{Q c}_{c^{\prime}}=0 \\
& \Longleftrightarrow\left[\begin{array}{c|c}
0 & -U \\
\hline 0 & I_{n-a}
\end{array}\right] c^{\prime}=0 \\
& c^{\prime}=\left[\begin{array}{c}
c_{a}^{\prime} \\
c_{n-a}^{\prime}
\end{array}\right]\left[\begin{array}{c|c}
0 & -U \\
\hline & \Longleftrightarrow
\end{array}\right]\left[\begin{array}{c}
c_{a}^{\prime} \\
c_{n-a}^{\prime}
\end{array}\right]=0 \\
& \Longleftrightarrow\left[\begin{array}{c}
-U c_{n-a}^{\prime} \\
c_{n-a}^{\prime}
\end{array}\right]=0 \\
& \Longleftrightarrow c_{n-a}^{\prime}=0 .
\end{aligned}
$$

Furthermore, we conclude that $c=A A^{(1)} c \Leftrightarrow c_{n-a}^{\prime}=0$.

Theorem 2. The vector

$$
x=A^{(1)} c+\left(I-A^{(1)} A\right) y,
$$

$y \in \mathbb{C}^{n \times 1}$ is an arbitrary column, is the general solution of the system (1), if and only if the $\{1\}$-inverse $A^{(1)}$ of the system matrix $A$ has the form (3) for arbitrary matrices $U$ and $W$ and the rows of the matrix $V\left(c_{a}^{\prime}-y_{a}^{\prime}\right)+y_{(n-a)}^{\prime}$ are free parameters, where $Q c=c^{\prime}=\left[c_{a}^{\prime} / 0\right]^{T}$ and $P^{-1} y=y^{\prime}=\left[y_{a}^{\prime} / y_{n-a}^{\prime}\right]^{T}$.

Proof. Since $\{1\}$-inverse $A^{(1)}$ of the matrix $A$ has the form (3), the solution of the system $x=A^{(1)} c+\left(I-A^{(1)} A\right) y$ can be represented in the form

$$
\begin{aligned}
x & =P\left[\begin{array}{c|c}
I_{a} & U \\
\hline V & W
\end{array}\right] Q c+\left(I-P\left[\begin{array}{c|c}
I_{a} & U \\
\hline V & W
\end{array}\right] Q A\right) y \\
& =P\left[\begin{array}{c|c}
I_{a} & U \\
\hline V & W
\end{array}\right] c^{\prime}+\left(I-P\left[\begin{array}{c|c}
I_{a} & U \\
\hline V & W
\end{array}\right] Q A P P^{-1}\right) y .
\end{aligned}
$$

According to Lemma 1 and from (2), we have

$$
x=P\left[\begin{array}{c|c}
I_{a} & U \\
\hline V & W
\end{array}\right]\left[\begin{array}{c}
c_{a}^{\prime} \\
\hline 0
\end{array}\right]+\left(I-P\left[\begin{array}{c|c}
I_{a} & U \\
\hline V & W
\end{array}\right]\left[\begin{array}{c|c}
I_{a} & 0 \\
\hline 0 & 0
\end{array}\right] P^{-1}\right) y .
$$

Furthermore, we obtain

$$
\begin{aligned}
& x=P\left[\begin{array}{c}
c_{a}^{\prime} \\
\hline V c_{a}^{\prime}
\end{array}\right]+\left(I-P\left[\begin{array}{c|c}
I_{a} & 0 \\
\hline V & 0
\end{array}\right] P^{-1}\right)\left[\begin{array}{c}
y_{a} \\
\hline y_{n-a}
\end{array}\right] \\
& =P\left[\begin{array}{c}
c_{a}^{\prime} \\
\hline V c_{a}^{\prime}
\end{array}\right]+\left(P P^{-1}-P\left[\begin{array}{c|c}
I_{a} & 0 \\
\hline V & 0
\end{array}\right] P^{-1}\right)\left[\begin{array}{c}
y_{a} \\
\hline y_{n-a}
\end{array}\right] \\
& =P\left[\begin{array}{c}
c_{a}^{\prime} \\
\hline V c_{a}^{\prime}
\end{array}\right]+P\left(I-\left[\begin{array}{c|c}
I_{a} & 0 \\
\hline V & 0
\end{array}\right]\right) P^{-1}\left[\frac{y_{a}}{y_{n-a}}\right] \\
& =P\left[\frac{c_{a}^{\prime}}{V c_{a}^{\prime}}\right]+P\left[\begin{array}{c|c}
0 & 0 \\
\hline-V & I_{n-a}
\end{array}\right]\left[\begin{array}{c}
y_{a}^{\prime} \\
\hline y_{n-a}^{\prime}
\end{array}\right],
\end{aligned}
$$

where $y^{\prime}=P^{-1} y$. We now conclude that

$$
\begin{aligned}
x & =P\left(\left[\frac{c_{a}^{\prime}}{V c_{a}^{\prime}}\right]+\left[\frac{0}{-V y_{a}^{\prime}+y_{n-a}^{\prime}}\right]\right) \\
& =P\left[\frac{c_{a}^{\prime}}{V\left(c_{a}^{\prime}-y_{a}^{\prime}\right)+y_{n-a}^{\prime}}\right] .
\end{aligned}
$$

Therefore, since matrix $P$ is regular, we deduce that $P\left[c_{a}^{\prime} /\left(V\left(c_{a}^{\prime}-y_{a}^{\prime}\right)+y_{n-a}^{\prime}\right)\right]^{T}$ is the general solution of the system (1) if and only if the rows of the matrix $V\left(c_{a}^{\prime}-y_{a}^{\prime}\right)+y_{n-a}^{\prime}$ are $n-a$ free parameters.

Corollary 3. The vector

$$
x=\left(I-A^{(1)} A\right) y,
$$

$y \in \mathbb{C}^{n \times 1}$ is an arbitrary column, is the general solution of the homogeneous linear system $A x=0, A \in \mathbb{C}^{m \times n}$, if and only if the $\{1\}$-inverse $A^{(1)}$ of the system matrix $A$ has the form (3) for arbitrary matrices $U$ and $W$ and the rows of the matrix $-V y_{a}^{\prime}+$ $y_{(n-a)}^{\prime}$ are free parameters, where $P^{-1} y=y^{\prime}=\left[y_{a}^{\prime} / y_{n-a}^{\prime}\right]^{T}$.

Example 4. Consider the homogeneous linear system

$$
\begin{aligned}
x_{1}+2 x_{2}+3 x_{3} & =0 \\
4 x_{1}+5 x_{2}+6 x_{3} & =0 .
\end{aligned}
$$

The system matrix is

$$
A=\left[\begin{array}{lll}
1 & 2 & 3 \\
4 & 5 & 6
\end{array}\right]
$$

For regular matrices

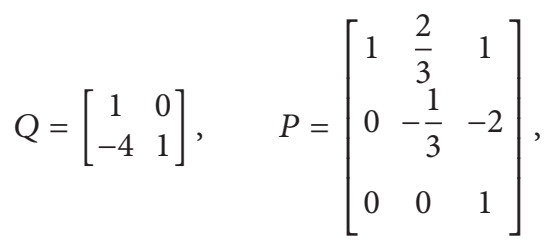


equality (2) holds. Rohde's general $\{1\}$-inverse $A^{(1)}$ of the system matrix $A$ is of the form

$$
A^{(1)}=P\left[\begin{array}{cc}
1 & 0 \\
0 & 1 \\
v_{11} & v_{12}
\end{array}\right] Q \text {. }
$$

According to Corollary 3 the general solution of the system (13) is of the form

$$
x=P\left[\begin{array}{cc|c}
0 & 0 & 0 \\
0 & 0 & 0 \\
\hline-v_{11} & -v_{12} & 1
\end{array}\right] P^{-1}\left[\begin{array}{l}
y_{1} \\
y_{2} \\
y_{3}
\end{array}\right],
$$

where

$$
P^{-1}=\left[\begin{array}{ccc}
1 & 2 & 3 \\
0 & -3 & -6 \\
0 & 0 & 1
\end{array}\right]
$$

Therefore, we obtain

$$
\begin{aligned}
x & =P\left[\begin{array}{cc|c}
0 & 0 & 0 \\
0 & 0 & 0 \\
\hline-v_{11}-v_{12} & 1
\end{array}\right]\left[\begin{array}{c}
y_{1}+2 y_{2}+3 y_{3} \\
-3 y_{2}-6 y_{3} \\
y_{3}
\end{array}\right] \\
& =P\left[\begin{array}{c}
0 \\
0 \\
-v_{11} y_{1}-\left(2 v_{11}-3 v_{12}\right) y_{2}-\left(3 v_{11}-6 v_{12}-1\right) y_{3}
\end{array}\right] .
\end{aligned}
$$

If we take $\alpha=-v_{11} y_{1}-\left(2 v_{11}-3 v_{12}\right) y_{2}-\left(3 v_{11}-6 v_{12}-1\right) y_{3}$ as a parameter, we get the general solution

$$
x=\left[\begin{array}{ccc}
1 & \frac{2}{3} & 1 \\
0 & -\frac{1}{3} & -2 \\
0 & 0 & 1
\end{array}\right]\left[\begin{array}{l}
0 \\
0 \\
\alpha
\end{array}\right]=\left[\begin{array}{c}
\alpha \\
-2 \alpha \\
\alpha
\end{array}\right]
$$

Corollary 5. The vector

$$
x=A^{(1)} c
$$

is the general solution of the system (1), if and only if the $\{1\}$-inverse $A^{(1)}$ of the system matrix $A$ has the form (3) for arbitrary matrices $U$ and $W$ and the rows of the matrix $V c_{a}^{\prime}$ are free parameters, where $Q c=c^{\prime}=\left[c_{a}^{\prime} / 0\right]^{T}$.

Remark 6. Similar result can be found in the paper by Malešević and Radičić [6].

Example 7. Consider the nonhomogeneous linear system

$$
\begin{gathered}
x_{1}+2 x_{2}+3 x_{3}=7 \\
4 x_{1}+5 x_{2}+6 x_{3}=8
\end{gathered}
$$

According to Corollary 5, the general solution of the system (22) is of the form

$$
x=P\left[\begin{array}{cc}
1 & 0 \\
0 & 1 \\
v_{11} & v_{12}
\end{array}\right] Q\left[\begin{array}{l}
7 \\
8
\end{array}\right]=P\left[\begin{array}{c}
7 \\
-20 \\
7 v_{11}-20 v_{12}
\end{array}\right] .
$$

If we take $\alpha=7 v_{11}-20 v_{12}$ as a parameter, we obtain the general solution of the system

$$
x=P\left[\begin{array}{c}
7 \\
-20 \\
\alpha
\end{array}\right]=\left[\begin{array}{ccc}
1 & \frac{2}{3} & 1 \\
0 & -\frac{1}{3} & -2 \\
0 & 0 & 1
\end{array}\right]\left[\begin{array}{c}
7 \\
-20 \\
\alpha
\end{array}\right]=\left[\begin{array}{c}
-\frac{19}{3}+\alpha \\
\frac{20}{3}-2 \alpha \\
\alpha
\end{array}\right]
$$

We are now concerned with the matrix equation

$$
A X=C,
$$

where $A \in \mathbb{C}^{m \times n}, X \in \mathbb{C}^{n \times k}$, and $C \in \mathbb{C}^{m \times k}$.

Lemma 8. The matrix equation (25) has a solution if and only if the last $m-$ a rows of the matrix $C^{\prime}=Q C$ are zeros, where $Q \in \mathbb{C}^{m \times m}$ is regular matrix such that (2) holds.

Proof. If we write $X=\left[\begin{array}{llll}X_{\downarrow 1} & X_{\downarrow 2} & \cdots & X_{\downarrow k}\end{array}\right]$ and $C=$ $\left[\begin{array}{llll}C_{\downarrow 1} & C_{\downarrow 2} & \cdots & C_{\downarrow k}\end{array}\right]$, then we can observe the matrix equation (25) as the system of matrix equations

$$
\begin{gathered}
A X_{\downarrow 1}=C_{\downarrow 1} \\
A X_{\downarrow 2}=C_{\downarrow 2} \\
\vdots \\
A X_{\downarrow k}=C_{\downarrow k} .
\end{gathered}
$$

Each of the matrix equation $A X_{\downarrow i}=C_{\downarrow i}, 1 \leq i \leq k$, by Lemma 1 has solution if and only if the last $m-a$ coordinates of the vector $C_{\downarrow i}^{\prime}=Q C_{\downarrow i}$ are zeros. Thus, the previous system has solution if and only if the last $m-a$ rows of the matrix $C^{\prime}=Q C$ are zeros, which establishes that matrix equation (25) has solution if and only if all entries of the last $m-a$ rows of the matrix $C^{\prime}$ are zeros.

Theorem 9. The matrix

$$
X=A^{(1)} C+\left(I-A^{(1)} A\right) Y \in \mathbb{C}^{n \times k},
$$

$Y \in \mathbb{C}^{n \times k}$ is an arbitrary matrix, is the general solution of the matrix equation (25) if and only if the $\{1\}$-inverse $A^{(1)}$ of the system matrix $A$ has the form (3) for arbitrary matrices $U$ and $W$ and the entries of the matrix

$$
V\left(C_{a}^{\prime}-Y_{a}^{\prime}\right)+Y_{(n-a)}^{\prime}
$$

are mutually independent free parameters, where $Q C=C^{\prime}=$ $\left[C_{a}^{\prime} / 0\right]^{T}$ and $P^{-1} Y=Y^{\prime}=\left[Y_{a}^{\prime} / Y_{n-a}^{\prime}\right]^{T}$.

Proof. Applying Theorem 2 on each system $A X_{\downarrow i}=C_{\downarrow i}, 1 \leq$ $i \leq k$, we obtain that

$$
X_{\downarrow i}=P\left[\frac{C_{a \downarrow i}^{\prime}}{V\left(C_{a \downarrow i}^{\prime}-Y_{a \downarrow i}^{\prime}\right)+Y_{n-a \downarrow i}^{\prime}}\right]
$$


is the general solution of the system if and only if the rows of the matrix $V\left(C_{a \downarrow i}^{\prime}-Y_{a \downarrow i}^{\prime}\right)+Y_{n-a \downarrow i}^{\prime}$ are $n-a$ free parameters. Assembling these individual solutions together we get that

$$
X=P\left[\frac{C_{a}^{\prime}}{V\left(C_{a}^{\prime}-Y_{a}^{\prime}\right)+Y_{n-a}^{\prime}}\right]
$$

is the general solution of matrix equation (25) if and only if entries of the matrix $V\left(C_{\mathrm{a}}^{\prime}-Y_{a}^{\prime}\right)+Y_{n-a}^{\prime}$ are $(n-a) k$ mutually independent free parameters.

From now on we proceed with the study of the nonhomogeneous linear system of the form

$$
x B=d,
$$

where $B$ is an $n \times m$ matrix over the field $\mathbb{C}$ of rank $b$ and $d$ is a $1 \times m$ matrix over $\mathbb{C}$. Let $R \in \mathbb{C}^{n \times n}$ and let $S \in \mathbb{C}^{m \times m}$ be regular matrices such that

$$
R B S=E_{b}=\left[\begin{array}{l|l}
I_{b} & 0 \\
\hline 0 & 0
\end{array}\right]
$$

An $\{1\}$-inverse of the matrix $B$ can be represented in Rohde's form

$$
B^{(1)}=S\left[\begin{array}{c|c}
I_{b} & M \\
\hline N & K
\end{array}\right] R
$$

where $M=\left[u_{i j}\right], N=\left[v_{i j}\right]$, and $K=\left[w_{i j}\right]$ are arbitrary matrices of corresponding dimensions $b \times(n-b),(m-b) \times b$ and $(m-b) \times(n-b)$ with mutually independent entries.

Lemma 10. The nonhomogeneous linear system (31) has a solution if and only if the last $m-b$ elements of the row $d^{\prime}=d S$ are zeros, where $S \in \mathbb{C}^{m \times m}$ is regular matrix such that (32) holds.

Proof. By transposing the system (31), we obtain system $B^{T} x^{T}=d^{T}$ and by transposing matrix equation (32) we obtain that $S^{T} B^{T} R^{T}=E_{b}$. According to Lemma 1, the system $B^{T} x^{T}=d^{T}$ has solution if and only if the last $m-b$ coordinates of the vector $S^{T} d^{T}$ are zeros, that is, if and only if the last $m-b$ elements of the row $d^{\prime}=d S$ are zeros.

Theorem 11. The row

$$
x=d B^{(1)}+y\left(I-B B^{(1)}\right) \text {, }
$$

$y \in \mathbb{C}^{1 \times n}$ is an arbitrary row, is the general solution of the system (31), if and only if the $\{1\}$-inverse $B^{(1)}$ of the system matrix $B$ has the form (33) for arbitrary matrices $N$ and $K$ and the columns of the matrix $\left(d_{b}^{\prime}-y_{b}^{\prime}\right) M+y_{n-b}^{\prime}$ are free parameters, where $d S=d^{\prime}=\left[\begin{array}{lll}d_{b}^{\prime} & \mid & 0\end{array}\right]$ and $y R^{-1}=y^{\prime}=\left[\begin{array}{lll}y_{b}^{\prime} & \mid & y_{n-b}^{\prime}\end{array}\right]$.

Proof. The basic idea of the proof is to transpose the system (31) and to apply Theorem 2 . The $\{1\}$-inverse of the matrix
$B^{T}$ is equal to a transpose of the $\{1\}$-inverse of the matrix $B$. Hence, we have

$$
\begin{aligned}
\left(B^{T}\right)^{(1)}=\left(B^{(1)}\right)^{T} & =\left(S\left[\begin{array}{c|c}
I_{b} & M \\
\hline N & K
\end{array}\right] R\right)^{T} \\
& =R^{T}\left[\begin{array}{c|c}
I_{b} & N^{T} \\
\hline M^{T} & K^{T}
\end{array}\right] S^{T} .
\end{aligned}
$$

We can now proceed analogously to the proof of Theorem 2 to obtain that

$$
x^{T}=R^{T}\left[\frac{d_{b}^{\prime T}}{M^{T}\left(d_{b}^{\prime T}-y_{b}^{\prime T}\right)+y_{n-b}^{\prime}{ }^{T}}\right]
$$

is the general solution of the system $B^{T} x^{T}=d^{T}$ if and only if the rows of the matrix $M^{T}\left(d_{b}^{\prime T}-y_{b}^{\prime T}\right)+y_{n-b}^{\prime}{ }^{T}$ are $n-b$ free parameters. Therefore,

$$
x=\left[d_{b}^{\prime} \mid\left(d_{b}^{\prime}-y_{b}^{\prime}\right) M+y_{n-b}^{\prime}\right] R
$$

is the general solution of the system (31) if and only if the columns of the matrix $\left(d_{b}^{\prime}-y_{b}^{\prime}\right) M+y_{n-b}^{\prime}$ are $n-b$ free parameters. Analogous corollaries hold for Theorem 11.

We now deal with the matrix equation

$$
X B=D,
$$

where $X \in \mathbb{C}^{k \times n}, B \in \mathbb{C}^{n \times m}$, and $D \in \mathbb{C}^{k \times m}$.

Lemma 12. Matrix equation (38) has a solution if and only if the last $m-b$ columns of the matrix $D^{\prime}=D S$ are zeros, where $S \in \mathbb{C}^{m \times m}$ is regular matrix such that (32) holds.

Theorem 13. The matrix

$$
X=D B^{(1)}+Y\left(I-B B^{(1)}\right) \in \mathbb{C}^{k \times n},
$$

$Y \in \mathbb{C}^{k \times n}$ is an arbitrary matrix, is the general solution of the matrix equation (38) if and only if the $\{1\}$-inverse $B^{(1)}$ of the system matrix $B$ has the form (33) for arbitrary matrices $N$ and $K$ and the entries of the matrix

$$
\left(D_{b}^{\prime}-Y_{b}^{\prime}\right) M+Y_{(n-b)}^{\prime}
$$

are mutually independent free parameters, where $D S=D^{\prime}=$ $\left[\begin{array}{l|l}D_{b}^{\prime} & 0\end{array}\right]$ and $Y R^{-1}=Y^{\prime}=\left[Y_{b}^{\prime} \mid Y_{n-b}^{\prime}\right]$.

\section{An Application}

In this section we will briefly sketch properties of the general solution of matrix equation

$$
A X B=C,
$$

where $A \in \mathbb{C}^{m \times n}, X \in \mathbb{C}^{n \times k}, B \in \mathbb{C}^{k \times l}$, and $C \in \mathbb{C}^{m \times l}$. If we denote by $Y$ matrix product $X B$, then the matrix equation (41) becomes

$$
A Y=C
$$


According to Theorem 9, the general solution of the system (42) can be presented as a product of the matrix $P$ and the matrix which has the first $a=\operatorname{rank}(A)$ rows same as the matrix $Q C$ and the elements of the last $m-a$ rows are $(m-a) n$ mutually independent free parameters; $P$ and $Q$ are regular matrices such that $Q A P=E_{a}$. Thus, we are now turning on to the system of the form

$$
X B=D .
$$

By Theorem 13, we conclude that the general solution of the system (43) can be presented as a product of the matrix which has the first $b=\operatorname{rank}(B)$ columns equal to the first $b$ columns of the matrix $D S$ and the rest of the columns have mutually independent free parameters as entries, and the matrix $R$, for regular matrices $R$ and $S$ such that $R B S=E_{b}$. Therefore, the general solution of the system (41) is of the form

$$
X=P\left[\begin{array}{c|c}
G_{a b} & F \\
\hline H & L
\end{array}\right] R,
$$

where $G_{a b}$ is a submatrix of the matrix QCS corresponding to the first $a$ rows and the first $b$ columns and the entries of the matrices $F, H$, and $L$ are $n k-a b$ free parameters. We will illustrate this on the following example.

Example 14. We consider the matrix equation

$$
A X B=C,
$$

where $A=\left[\begin{array}{cc}1 & -2 \\ -2 & 4\end{array}\right], B=\left[\begin{array}{lll}1 & 2 & 1 \\ 1 & 2 & 1 \\ 1 & 2\end{array}\right]$ and $C=\left[\begin{array}{ccc}1 & 2 & 1 \\ -2 & -4 & -2\end{array}\right]$. If we take $Y=X B$, we obtain the system

$$
A Y=C .
$$

It is easy to check that the matrix $A$ is of the rank $a=1$ and for matrices $Q=\left[\begin{array}{ll}1 & 0 \\ 2 & 1\end{array}\right]$ and $P=\left[\begin{array}{ll}1 & 2 \\ 0 & 1\end{array}\right]$ the equality $Q A P=$ $E_{a}$ holds. Based on Theorem 9, the equation $A Y=C$ can be rewritten in the system form

$$
\begin{aligned}
& A Y_{\downarrow 1}=\left[\begin{array}{c}
1 \\
-2
\end{array}\right], \\
& A Y_{\downarrow 2}=\left[\begin{array}{c}
2 \\
-4
\end{array}\right], \\
& A Y_{\downarrow 3}=\left[\begin{array}{c}
1 \\
-2
\end{array}\right] .
\end{aligned}
$$

Combining Theorem 2 with the equality

$$
\left[\begin{array}{ccc}
c_{1}^{\prime} & c_{2}^{\prime} & c_{3}^{\prime} \\
0 & 0 & 0
\end{array}\right]=\left[\begin{array}{ll}
1 & 0 \\
2 & 1
\end{array}\right]\left[\begin{array}{ccc}
1 & 2 & 1 \\
-2 & -4 & -2
\end{array}\right]=\left[\begin{array}{lll}
1 & 2 & 1 \\
0 & 0 & 0
\end{array}\right]
$$

yields

$$
\begin{aligned}
& Y_{\downarrow 1}=P[\underbrace{v-v z_{11}+z_{21}}_{\alpha}], \\
& Y_{\downarrow 2}=P[\underbrace{2 v-2 v z_{12}+z_{22}}_{\beta}], \\
& Y_{\downarrow 3}=P[\underbrace{v-v z_{13}+z_{23}}_{\gamma}],
\end{aligned}
$$

for an arbitrary matrix $Z=\left[\begin{array}{lll}z_{11} & z_{12} & z_{13} \\ z_{21} & -z_{22} & z_{23}\end{array}\right]$. Therefore, the general solution of the system $A Y=C$ is

$$
Y=P\left[\begin{array}{lll}
1 & 2 & 1 \\
\alpha & \beta & \gamma
\end{array}\right]
$$

From now on, we consider the system

$$
X B=D
$$

for

$$
D=P\left[\begin{array}{ccc}
1 & 2 & 1 \\
\alpha & \beta & \gamma
\end{array}\right]=\left[\begin{array}{ccc}
1+2 \alpha & 2+2 \beta & 1+2 \gamma \\
\alpha & \beta & \gamma
\end{array}\right] .
$$

There are regular matrices $R=\left[\begin{array}{ccc}1 & 0 & 0 \\ -1 & 1 & 0 \\ -1 & 0 & 1\end{array}\right]$ and $S=\left[\begin{array}{ccc}1 & -2 & -1 \\ 0 & 1 & 0 \\ 0 & 0 & 1\end{array}\right]$ such that $R B S=E_{b}$ holds. Since the rank of the matrix $B$ is $b=1$, according to Lemma 12 all entries of the last two columns of the matrix $D^{\prime}=D S$ are zeros; that is, we have $\gamma=\alpha, \beta=2 \alpha$. Hence, we get that the matrix $D^{\prime}$ is of the form $D^{\prime}=\left[\begin{array}{ccc}1+2 \alpha & 0 & 0 \\ \alpha & 0 & 0\end{array}\right]$. Applying Theorem 13, we obtain

$$
X=\left[\begin{array}{ccc}
1+2 \alpha & \underbrace{\left(1+2 \alpha-t_{11}\right) m_{11}+t_{12}}_{\gamma_{1}} & \underbrace{\left(1+2 \alpha-t_{11}\right) m_{12}+t_{13}}_{\beta 1} \\
\alpha & \underbrace{\left(\alpha-t_{21}\right) m_{11}+t_{22}}_{\gamma_{1}} & \underbrace{\left(\alpha-t_{12}\right) m_{12}+t_{23}}_{\gamma_{2}}
\end{array}\right] R \text {, }
$$

for an arbitrary matrix $T=\left[\begin{array}{ccc}t_{11} & t_{12} & t_{13} \\ t_{21} & t_{22} & t_{23}\end{array}\right]$. Finally, the solution of the system $A X B=C$ is

$$
X=\left[\begin{array}{ccc}
1+2 \alpha-\beta_{1}-\beta_{2} & \beta_{1} & \beta_{2} \\
\alpha-\gamma_{1}-\gamma_{2} & \gamma_{1} & \gamma_{2}
\end{array}\right] .
$$

\section{Acknowledgment}

This research is partially supported by the Ministry of Science and Education of Serbia, Grant no. 174032.

\section{References}

[1] A. Ben-Israel and T. N. E. Greville, Generalized Inverses: Theory and Applications, Springer, New York, NY, USA, 2nd edition, 2003.

[2] S. L. Campbell and C. D. Meyer, Generalized Inverses of Linear Transformations, SIAM Series Classics in Applied Mathematics, Philadelphia, Pa, USA, 2009. 
[3] C. A. Rohde, Contribution to the theory, computation and application of generalized inverses [Doctoral Dissertation], University of North Carolina at Raleigh, 1964.

[4] V. Perić, "Generalized reciprocals of matrices," Matematika, vol. 11, no. 1, pp. 40-57, 1982 ().

[5] N. S. Urquhart, "The nature of the lack of uniqueness of generalized inverse matrices," SIAM Review, vol. 11, pp. 268-271, 1969.

[6] B. Malešević and B. Radičić, "Non-reproductive and reproductive solutions of some matrix equations," in Proceedings of International Conference in Mathematical and Informational Technologies, pp. 246-2251, Vrnjačka Banja, Serbia, 2011.

[7] B. Malešević and B. Radičić, "Reproductive and nonreproductive solutions of the matrix equation $\mathrm{AXB}=\mathrm{C}$," in Proceedings of the 2nd Symposium on Mathematics and Applications, pp. 157-163, Faculty of Mathematics, Belgrade, Serbia, 2011.

[8] B. Malešević and B. Radičić, "Some considerations of matrix equations using the concept of reproductivity," Kragujevac Journal of Mathematics, vol. 36, no. 1, pp. 151-161, 2012.

[9] R. Penrose, "A generalized inverse for matrices," Mathematical Proceedings of the Cambridge Philosophical Society, vol. 51, pp. 406-413, 1955. 


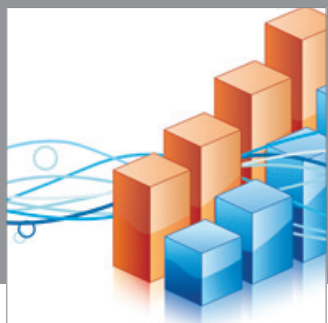

Advances in

Operations Research

mansans

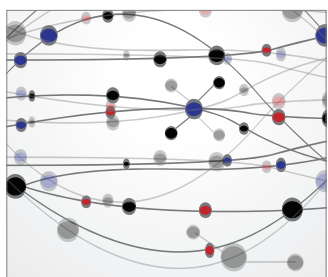

The Scientific World Journal
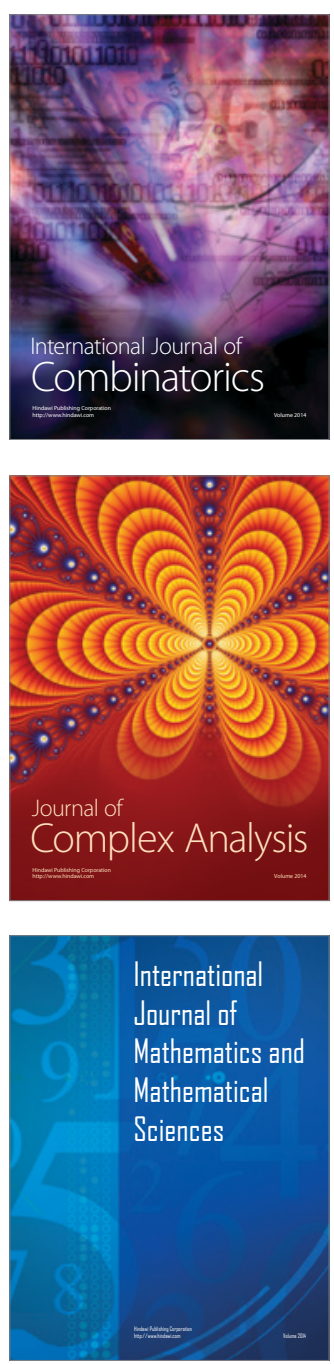
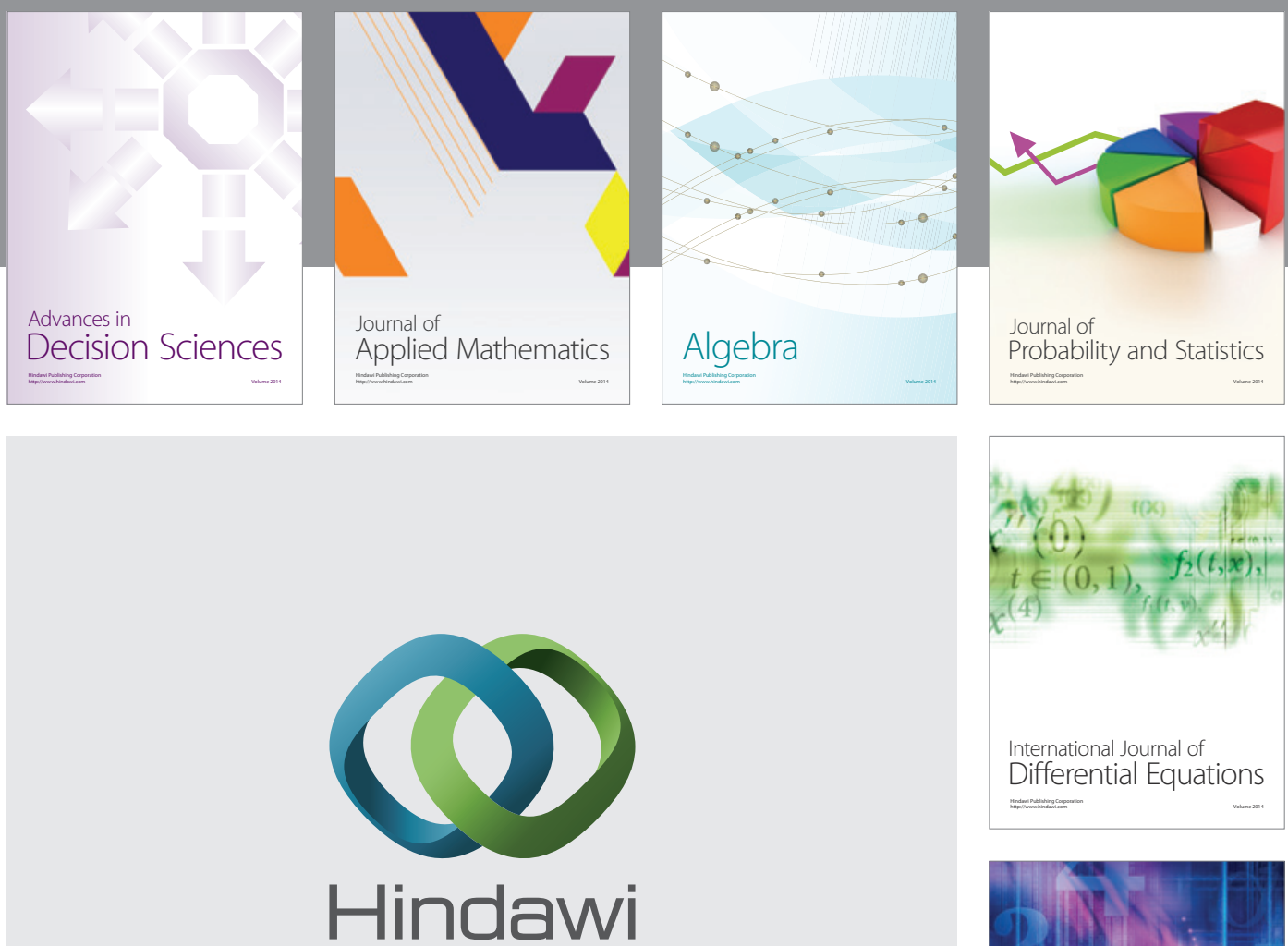

Submit your manuscripts at http://www.hindawi.com
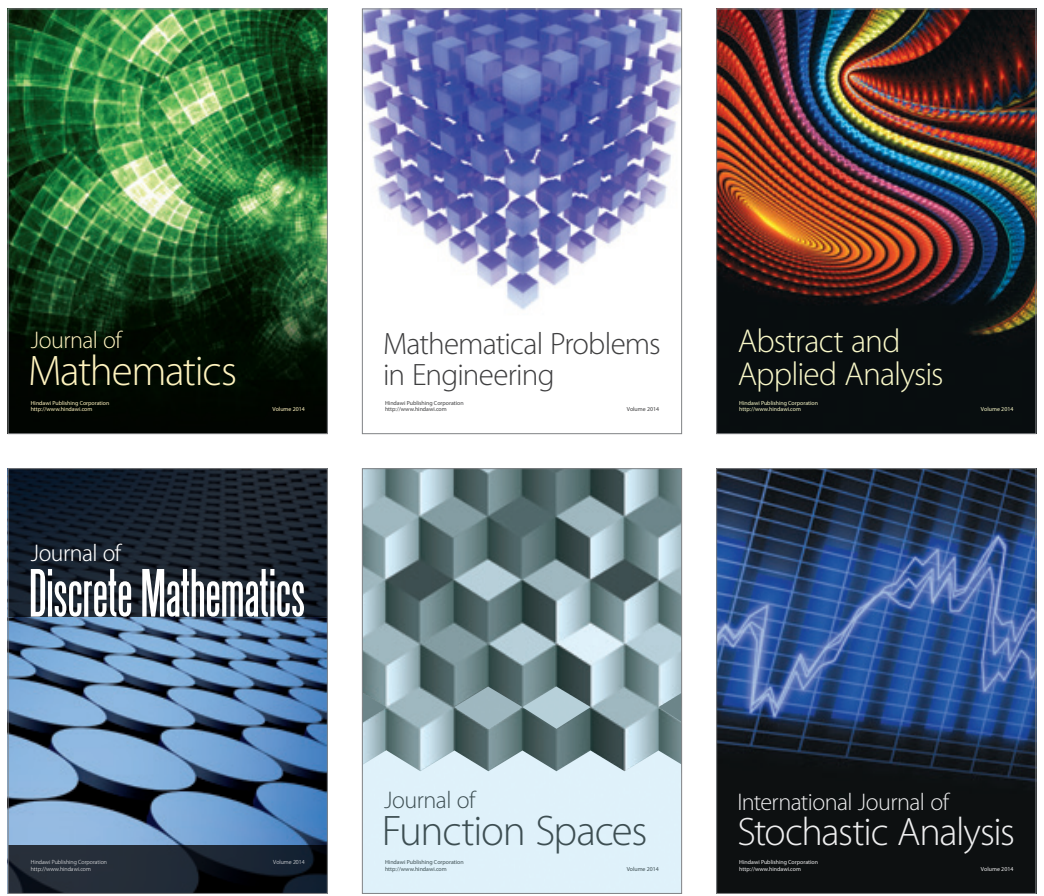

Journal of

Function Spaces

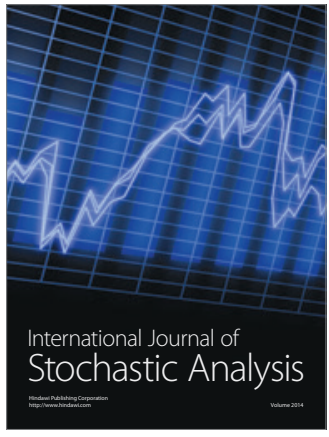

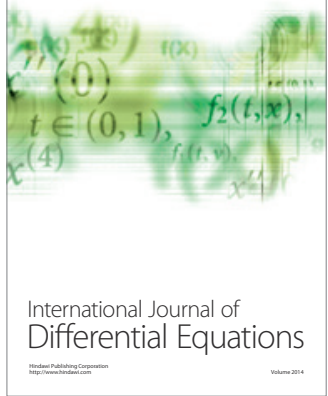
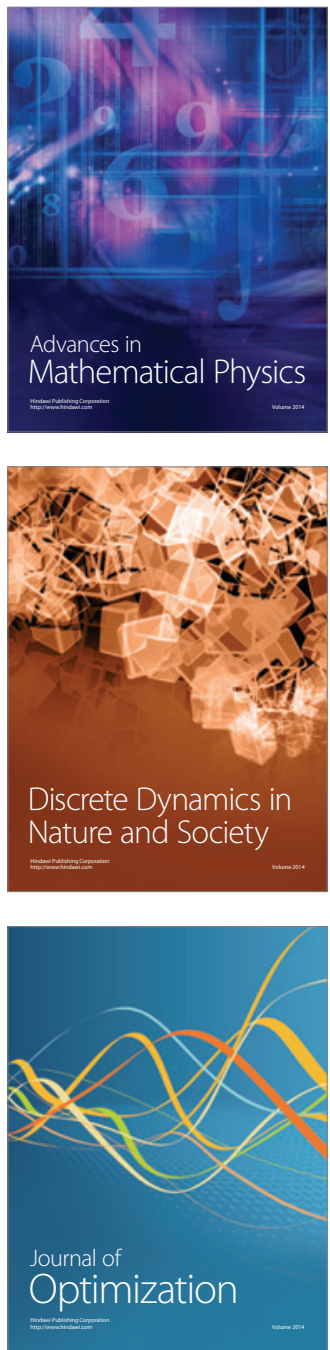